\title{
CONCERNING SIMPLE PLANE WEBS
}

\author{
BY
}

R. H. BING

A compact continuum $W$ is said [1](1) to be a simple web if there exists an upper semi-continuous collection $G$ of mutually exclusive continua filling up $W$ and another such collection $H$ also filling up $W$ such that (1) $G$ is a dendron with respect to its elements and so is $H$ and (2) if $g$ and $h$ are elements of $G$ and $H$, respectively, the common part of $g$ and $h$ exists and is totally disconnected. Hence, a simple web is a web $\left({ }^{2}\right)$.

Examples of simple plane webs. Although a simple web is not necessarily a subset of the plane, this paper will deal only with those of this type. A square plus its interior is a simple web. We may consider the elements of $G$ to be intervals parallel to one pair of sides of the square and the elements of $H$ to be intervals parallel to the other pair of sides. We see from Theorem 1 of this paper that if $C_{1}, C_{2}, \ldots$ are circles no two of which intersect each other and such that $C_{1}$ incloses $C_{i}(i=2,3, \cdots)$, then $C_{1}$ plus its interior minus the sum of the interiors of $C_{2}, C_{3}, \cdots$ is a simple web.

A square plus its interior plus an interval intersecting both the interior and the exterior of the square is not a simple web. Hence, a plane web is not necessarily a simple web. By the use of Theorem 1 of this paper, we find that the continuous curve described on page 273 of [7] which is left connected but not locally connected on the omission of some countable subset is not a simple web.

This paper gives necessary and sufficient conditions that a compact plane continuum be a simple plane web. Professor R. L. Moore suggested the problem of finding such conditions. Much credit is due him for the development of this paper.

It has been shown [6] that every simple plane web is a continuous curve. In the present paper, the following theorem will be established.

THEOREM 1. In order that a compact plane continuous curve be a simple plane web, it is necessary and sufficient that it remain connected and locally connected on the omission of any countable subset.

Presented to the Sociéty, September 17, 1945; received by the editors May 12, 1945 and, in revised form, December 24, 1945.

(1) Numbers in brackets refer to the references cited at the end of the paper.

(2) A web has been defined by Moore [3] to be a compact continuum $M$ for which "there exist two upper semicontinuous collections $H_{1}$ and $H_{2}$ of mutually exclusive continua such that (1) each of these collections fills up $M$, (2) each of them is a dendron with respect to its elements, and (3) there exists an uncountable subcollection $W$ of the collection $H_{1}$ such that no element of $W$ is a subset of any element of $H_{2}$." 
Proof of necessity. The following argument is similar to that found in the proof of Theorem 1 of [1]. Suppose that $G$ and $H$ are collections satisfying with respect to the simple plane web $W$ all $\left.{ }^{3}\right)$ of the conditions described in the preceding definition. Suppose that $K$ is a countable subset of $W$. If $W_{Z}$ $(Z=G, H)$ denotes the sum of all of the continua of the collection $Z$ that do not intersect $K$, then any two points of $W_{G}+W_{H}$ lie on a continuum which is the sum of three continua of the collection $G+H$ that do not intersect $K$. As $W_{G}+W_{H}$ is dense in $W, W-K$ is connected.

If $W-K$ is not locally connected at $P$, there exist a domain ( $\left.{ }^{4}\right) d$ containing $P$ and a sequence of points of $W-K$ converging to $P$ such that each belongs to a different component of $(W-K) \cdot d$. Let $C_{1}, C_{2}, C_{3}$ denote three circles such that $C_{1}$ incloses $P, C_{2}$ incloses $C_{1}, C_{3}$ incloses $C_{2}$ but no element of $G$ or $H$ and $C_{3}$ plus its interior is a subset of $d$. Now $C_{1}$ incloses a sequence of points $P_{1}, P_{2}, \cdots$ of $W_{G}$ no two of which belong to the same component of $(W-K) \cdot d$ such that if $g_{i}$ is the component containing $P_{i}$ of the common part of $d$ and the element of $G$ containing $P_{i}$, then $g_{1}, g_{2}, \ldots$ has a sequential limiting set $F$. There exists a sequence $Q_{1}, Q_{2}, \cdots$ in $W_{H}$ converging to a point of $C_{2}$ such that $Q_{n}$ is a point of a subcontinuum of $g_{m}(m \geqq n)$ irreducible from $C_{1}$ to $C_{3}$. If $h_{i}$ denotes the component containing $Q_{i}$ of the common part of $d$ and the element of $H$ containing $Q_{i}$, then some nondegenerate subcontinuum of $F$ belongs (see [6, Theorem 2]) to the limiting set of $h_{1}, h_{2}, \cdots$. However, as $G$ and $H$ are upper semi-continuous collections, this nondegenerate continuum is both a subset of an element of $G$ and a subset of an element of $H$. This is contrary to the hypothesis that the common part of an element of $G$ and an element of $H$ is totally disconnected.

This establishes the necessity of the condition of Theorem 1. A number of preliminary theorems will be proved before the establishment of its sufficiency. When the term "simple plane web" is used in the following theorems, it is to be understood that we refer to a compact plane continuum that remains connected and locally connected on the omission of any countable subset.

TheOREM 2. Each complementary domain of a simple plane web is bounded by a simple closed curve but no two boundaries of its individual complementary domains have more than one point in common.

Proof. The outer boundary of a bounded complementary domain of a continuous curve is a simple closed curve [4]. As a simple plane web has no cut points, the boundary of one of its complementary domains is also the outer boundary of this domain. If the boundaries of two such complementary

(3) However, in the argument no use is made of the condition that $G$ and $H$ are dendrons with respect to their elements.

(4) A domain is an open set not necessarily connected. However, a complementary domain is connected [7, pp. 11 and 153]. 
domains intersected in a pair of points, this pair of points would separate the simple plane web.

THEOREM 3. If the connected set $x$ is the sum of a countable number of points and complementary domains of the simple plane web $W$, then each point of $W \cdot \bar{x}$ is a limit point of $W-W \cdot \bar{x}$.

Proof. Suppose that $d$ is a domain containing a point $P$ of $W \cdot \bar{x}$. As $W-W \cdot x$ is a connected and locally connected inner limiting set, the common part of it and $d$ contains [8] an arc $A Q B$. There exists in $W$ an $\operatorname{arc} A C B$ containing no point of the countable point set $W \cdot x+Q$. The point set $A Q B+A C B$ contains a simple closed curve $J$ which contains an interval $A^{\prime} Q B^{\prime}$ of $A Q B$. There exists in $W$ an arc $\alpha$ from $Q$ to $J-A^{\prime} Q B^{\prime}$ which contains no point of $W \cdot x+A^{\prime}+B^{\prime}$. The arc $\alpha$ contains an arc $Q^{\prime} O L$ having in common with $J$ only its end points $Q^{\prime}$ and $L$ which belong to $A^{\prime} Q B^{\prime}$ and $J-A^{\prime} Q B^{\prime}$ respectively. Let $Q^{\prime} A^{\prime} L$ and $Q^{\prime} B^{\prime} L$ be arcs lying on $J$ with points as indicated. Then $Q^{\prime} A^{\prime} L+Q^{\prime} B^{\prime} L+Q^{\prime} O L$ divides space into three mutually exclusive domains only one of which contains the connected set $x$. Now one of the segments( $\left.{ }^{5}\right)\left\langle Q^{\prime} A^{\prime} L\right\rangle,\left\langle Q^{\prime} B^{\prime} L\right\rangle$ and $\left\langle Q^{\prime} O L\right\rangle$ contains no point of $\bar{x}$ and each of them contains a point of $d$. Therefore $d$ contains a point of $W-W \cdot \bar{x}$. Since any domain containing a point of $W \cdot \bar{x}$ contains a point of $W-W \cdot \bar{x}$, then each point of $W \cdot \bar{x}$ is a limit point of $W-W \cdot \bar{x}$.

THEOREM 4. Under the hypotheses of Theorem 3, the closure of $x$ does not separate $W$.

Proof. If $P$ is a point of the plane $S$, then $S-(x+P)$ is a connected and locally connected inner limiting set. Hence [8] it is arcwise connected. Furthermore [2, Theorem 12], $S-\bar{x}$ is connected.

Suppose that $W-W \cdot \bar{x}$ is the sum of two mutually separated sets $S_{1}$ and $S_{2}$. There exists in $S-\bar{x}$ an arc $A B$ from a point $A$ of $S_{1}$ to a point $B$.of $S_{2}$. Let $P$ denote the last point of the closed point set $S_{1} \cdot A B$ in the order from $A$ to $B$ on $A B$. There is a point $Q$ following $P$ on $A B$ such that the interval $P Q$ of $A B$ contains no point of $S_{2}$. Let $P^{\prime}$ denote the first point in the order from $Q$ to $B$ that the interval $Q B$ of $A B$ has in common with $\beta$, the boundary of $d$, the complementary domain of $W$ that contains $Q$. Since the closure of $x+d$ does not separate $S$, one arc of $\beta$ from $P$ to $P^{\prime}$ contains no point of $\bar{x}$. But $P$ belongs to $S_{1}$ and $P^{\prime}$ belongs to $S_{2}$. Thus, the supposition that $W-W \cdot \bar{x}$ is not connected leads to a contradiction.

THEOREM 5. If $R$ is a connected point set consisting of a plane domain plus a totally disconnected point set and the complement of $R$ is a locally connected inner limiting set, then $\bar{R}$ is a continuous curve.

Proof. If $\bar{R}$ is not locally connected, there exist a domain $d$, a sequence

(5) By $\langle\alpha\rangle$ is meant the $\operatorname{arc} \alpha$ minus its end points. 
of components $c_{1}, c_{2}, \cdots$ of $\bar{d} \cdot \bar{R}$, and a sequence of $\operatorname{arcs} \alpha_{1}, \alpha_{2}, \cdots$ such that (1) for each positive integer $j, \alpha_{j}$ is a subset of $\bar{d}-\bar{d} \cdot \bar{R}$; (2) if $h, i, j$ are positive integers such that $h<i<j$, then $\alpha_{i}$ separates $c_{h}$ from $c_{j}$ in $d$ and $c_{i}$ separates $\alpha_{k}$ from $\alpha_{j}$ in $d$; (3) $d$ intersects $c$, the sequential limiting set of $c_{1}, c_{2}, \cdots$. The sequence $\alpha_{1}, \alpha_{2}, \cdots$ converges to $c$.

As no point of $c$ is a point of a domain lying in $R$ and some nondegenerate subcontinuum $c^{\prime}$ of $c$ is a subset of $d$, the complement of $R$ contains two points $P_{1}$ and $P_{2}$ of $c^{\prime}$. There exist domains $d_{1}$ and $d_{2}$ containing $P_{1}$ and $P_{2}$ respectively such that $d_{1}$ and $d_{2}$ are mutually exclusive subsets of $d$. As the complement of $R$ is locally arcwise connected at $P_{1}$ and $P_{2}$, there exists a positive integer $j$ such that each of the point sets $d_{1}-d_{1} \cdot R$ and $d_{2}-d_{2} \cdot R$ contains an arc intersecting both $\alpha_{j}$ and $\alpha_{j+2}$. Therefore, there is a simple closed curve $J$ lying in $d-d \cdot R$ and intersecting $\alpha_{j}$ and $\alpha_{j+2}$. As $R$ is connected and contains no point of $J$, it does not intersect both the interior and the exterior of $J$. Therefore, no component of $\bar{d} \cdot \bar{R}$ separates $\alpha_{j}$ from $\alpha_{j+2}$ in $d$ and $\bar{R}$ is locally connected.

THEOREM 6. Under the hypotheses of Theorem $3, \bar{x}$ is a continuous curve each of whose degenerate cyclic elements is a point of $W$ in $x$ and each of whose nondegenerate cyclic elements is the closure of a complementary domain of $W$ in $x$.

Proof. As the complement of $x$ is a locally connected inner limiting set, it follows from Theorem 5 that $\bar{x}$ is a continuous curve. If $P$ is a cut point of $\bar{x}$, it is a cut point of $x$ and therefore must be a point of $W$ in $x$.

If $J$ is a simple closed curve in $\bar{x}$, we shall show that it is a subset of the closure of a complementary domain of $W$ in $x$. Since $\bar{x}$ does not separate space (see Theorem 4), one of the complementary domains of $J$ is a subset of $\bar{x}$. By Theorem 3, we have that $J$ separates no two points of $\bar{x}-x$ from each other. Therefore, one of the complementary domains of $J$ is a subset of $x$ and is therefore a subset of a complementary domain of $W$ in $x$.

Theorem 7. Suppose $W$ is a simple plane web, $\beta$ is its outer boundary and $X$ is the collection of all nondegenerate continua $x$ such that $x$ is maximal with respect to being the closure of a connected set which is the sum of a countable number of points and bounded complementary domains of $W$. If $A$ and $B$ are points of $W$, there is an arc $A B$ in $W$ such that $A B$ minus its end points contains no point of either $\beta$ or any element of $X$.

Proof. Suppose that $A$ belongs to $x_{A}$ of $X$ and that $B$ belongs to neither $\beta$ niour any element of $X$. As $W-W \cdot x_{A}+A$ is connected, $A$ is accessible [10, Theorem 1] from $W-W \cdot x_{A}$. Since the continua of the collection $X$ are mutually exclusive and no one of them separates the plane, then $x_{A}$ plus the complement of the sum of the elements of $X$ is a connected and locally connected inner limiting set which if regarded as space satisfies Axioms 1-5 of [7]. In this space, there exists an arc $A B$ having no point except $A$ in common with 
$x_{A}+\beta$. The $\operatorname{arc} A B$ is a subset of $W$.

THEOREM 8. Under the hypotheses of Theorem 7 , if $P$ is a point belonging to both $\beta$ and an element $x_{P}$ of $X, Q$ is another point of $\beta$ and $c$ is a component of $x_{P}-P$, then there exists in $W$ a simple closed curve $J$ inclosing $c$ but no point of $x_{P}-c$ and containing $P$ and $Q$ but no other point either of $\beta$ or of an element of $X$.

Proof. Let $X^{\prime}$ denote the set of all continua of the collection $X$ except $x_{P}$ and $x_{Q}$ where $x_{Q}$ denotes the element of $X$ containing $Q$ if there is one but denotes $Q$ if there is not. Let $Z$ denote the collection whose elements are the continua of $X^{\prime}$ and the points of the plane that belong to no element of $X^{\prime}$. The collection $Z$ is upper semi-continuous and no one of its elements separates the plane. It follows [5] that the space $\Sigma_{1}$ whose "points" are elements of $Z$ is topologically equivalent to the plane. If $\Sigma_{2}$ denotes the space whose "points" are the "points" of $\Sigma_{1}$ other than the elements of $X^{\prime}$ not intersecting $\beta$, then it is topologically equivalent to a plane minus a countable number of points. If $\beta^{\prime}$ denotes the "point" set whose points are the points of $\beta$ that belong to no element of $X^{\prime}$ and the continua of $X^{\prime}$ that intersect $\beta$, then in the space $\Sigma_{2}, \beta^{\prime}$ is a simple closed curve and $x_{P}$ and $x_{Q}$ are continuous curves each lying except for one point wholly in the interior of $\beta^{\prime}$. In this space, there exists an arc $E Q$ from a point $E$ of $c$ to $Q$ such that $x_{P}+x_{Q}+\beta^{\prime}$ intersects $E Q$ only at $E+Q$. Furthermore [7, Theorem 12, p. 217], there exists a simple closed curve intersecting $x_{P}+x_{Q}+\beta^{\prime}$ only at $P+Q$ and inclosing $c+E Q-Q$ but no point of $x_{P}-c$.

Description of dendrons $G$ and $F$. In this section, $W$ will designate a compact plane continuous curve which is left connected and locally connected on the omission of any countable point set. Also, $X$ will denote the set of all nondegenerate continua $x$ such that $x$ is maximal with respect to being the closure of a connected set which is the sum of a countable number of points and bounded complementary domains of $W$.

As a step toward proving the sufficiency of Theorem 1, we shall describe an upper semi-continuous collection $G$ of mutually exclusive continua filling up $W$ such that $G$ is a dendron with respect to its elements. At the same time, we shall describe a dendron $F$ in $W$ such that each element of $G$ intersects $F$ in only one point.

As the description of $G$ is somewhat complicated, we shall describe it by steps. First, we consider a transformation $T$ throwing a square plus a subset of its interior into $W$. Next, we describe an upper semi-continuous collection of mutually exclusive continua which is an arc with respect to its elements and which covers a part of the inverse of $W$ under transformation $T$. We cover more of this inverse by another upper semi-continuous collection of mutually exclusive continua. The process is continued until we have an upper semicontinuous collection of mutually exclusive continua filling up the inverse 
of $W$ under $T$ such that this collection is a dendron with respect to its elements. The image of the elements of this collection with respect to $T$ gives the elements of $G$.

Transformation $T$. Let $P_{1} P_{2} P_{3}$ and $P_{1} P_{4} P_{3}$ be two arcs whose sum is the boundary $\beta$ of the unbounded complementary domain of $W$ and whose ends are not points of $X^{*}\left({ }^{6}\right)$. There exist in $W$ arcs $P_{1} P_{5} P_{3}$ and $P_{1} P_{6} P_{3}$ which except for their end points are mutually exclusive and contain no point of $X^{*}+\beta$. Let $R$ be the interior of a square with diagonals $V_{1} V_{3}$ and $V_{2} V_{4}$ and let $V_{5}, V_{6}$ be the midpoints of $V_{1} V_{4}, V_{2} V_{3}$ respectively.

There exists a continuous transformation $T$ of $\bar{R}$ into $W+X^{*}$ such that $T\left(V_{1} V_{5}\right)=T\left(V_{4} V_{5}\right)=P_{1} P_{5}, T\left(V_{2} V_{6}\right)=T\left(V_{3} V_{6}\right)=P_{3} P_{6}, \quad T\left(V_{5} V_{2}\right)=P_{5} P_{3}$, $T\left(V_{4} V_{6}\right)=P_{1} P_{6}$ and each point of $W+X^{*}-P_{1} P_{5}-P_{6} P_{3}$ is the image of only one point of $\bar{R}-V_{1} V_{5} V_{4}-V_{2} V_{6} V_{3}$ under the transformation $T$.

Let $M$ denote the set of all points $P$ of $\bar{R}$ such that the image of $P$ under $T$ is a point of $W$. We note that $M$ is a compact continuous curve which is left connected and locally connected on the omission of any countable subset. Let $Y$ be the collection of all continua $y$ such that $y$ is the inverse under $T$ of an element of $X$. We note that $y$ is maximal with respect to being the closure of a connected set which is the sum of a countable number of points and bounded complementary domains of $M$.

The elements of $Y$ are ordered $y_{1}, y_{2}, \ldots$. By Theorem 7 and the fact that $M$ is separable, there exists a countable point set $z_{1}, z_{2}, \cdots$ dense in $M$ and such that for each positive integer $i, z_{i}$ belongs to $M$ but not to $Y^{*}$ or the boundary of $R$. The bounded complementary domains of $M$ are ordered $d_{1}, d_{2}, \cdots$.

We shall describe an upper semi-continuous collection $G^{\prime}$ of mutually exclusive continua filling up $M$ such that (1) $G^{\prime}$ is a dendron with respect to its elements, (2) no element of $G^{\prime}$ contains more than two points of $Y^{*}$, (3) each element of $G^{\prime}$ intersects $V_{5} V_{2}$ and $V_{4} V_{6}$, and (4) each element of $G^{\prime}$ is a continuum of condensation of $M$. Also, we shall describe a dendron $F^{\prime}$ in $M$ such that each element of $G^{\prime}$ intersects $F^{\prime}$ in only one point.

First step. If some element of $Y$ has a point $P$ in common with $V_{1} V_{2}$ or $V_{4} V_{3}$ and $P$ is nearer the midpoint than either end point of the one of $V_{1} V_{2}$, $V_{4} V_{3}$ on which it lies, let $i$ be the least positive integer $n$ such that $y_{n}$ is such an element of $Y$ and let $P$ be the point that $y_{i}$ has in common with $V_{1} V_{2}+V_{4} V_{3}$. If there is no such $i$, let $P$ be the midpoint of $V_{1} V_{2}$. Let $P_{1}$ and $P_{2}$ be the projections of $P$ on $V_{1} V_{2}$ and $V_{4} V_{3}$ respectively. Let $k$ be the least positive integer $n$, if there is one, such that $y_{n}$ has no point in common with the boundary of $R$. There are two arcs $P_{1} A P_{2}$ and $P_{1} D P_{2}$ lying in $M$, intersecting $Y^{*}$ plus the boundary of $R$ only at $P_{1}$ and $P_{2}$ and forming a simple closed curve $J$ such that (1) $J$ incloses $z_{1}$ and $y_{k},(2)$ the interior of $J$ together with $P_{1}$ and $P_{2}$ contains each element of $Y$ (if any) that contains

${ }^{(6)}$ By $X^{*}$ is meant the set of all points $P$ such that $P$ is a point of an element of $X$. 
$P_{1}$ or $P_{2}$, and (3) $P_{1} A P_{2}$ separates $\left\langle P_{1} D P_{2}\right\rangle$ from $V_{1} V_{4}$ in $M$.

We shall consider the case in which $P_{1}$ and $P_{2}$ belong to elements $y\left(P_{1}\right)$ and $y\left(P_{2}\right)$ of $Y$ respectively. Let $\left\langle P_{1} B P_{2}\right\rangle$ and $\left\langle P_{1} C P_{2}\right\rangle$ be mutually exclusive open arcs in $M$ which are inclosed by $J$, which contain no point of $Y^{*}$, and which are such that $P_{1} B P_{2}$ separates $\left\langle P_{1} C P_{2}\right\rangle$ from $V_{1} V_{4}$ in $M$, the simple closed curve formed by $P_{1} A P_{2}+P_{1} B P_{2}$ incloses $y\left(P_{1}\right)-P_{1}$, the simple closed curve formed by $P_{1} B P_{2}+P_{1} C P_{2}$ incloses $y\left(P_{2}\right)-P_{2}$, and $P_{2} C$ of $P_{1} C P_{2}$ intersects $V_{\mathbf{5}} V_{2}$.

If $y\left(P_{1}\right)-P_{1}$ is not connected, let $c_{1}, c_{2}, \cdots$ denote its components. Let $i, j$ be the least positive integers $m, n$ respectively such that $z_{m}, y_{n}$ are inclosed by $P_{1} A P_{2}+P_{1} B P_{2}$. There is a simple closed curve $J_{1}$ in $M$ containing $P_{1}$ and $P_{2}$ and inclosing $c_{1}, z_{i}, y_{j}$ but no point of $y\left(P_{1}\right)-c_{1}$ which is such that $J_{1}-P_{1}-P_{2}$ contains no point of $Y^{*}$ and is inclosed by $P_{1} A P_{2}+P_{1} B P_{2}$. Let $O$ represent the simple closed curve which is a subset of $P_{1} A P_{2}+P_{1} B P_{2}+J_{1}$ and which incloses $c_{2}$ but no point of $J_{1}$. Let $i, j$ be the least positive integers $m, n$ such that $z_{m}, y_{n}$ are inclosed by $O$. There is a simple closed curve $J_{2}$ in $M$ containing $P_{1}$ and $P_{2}$ and inclosing $c_{2}, z_{i}, y_{j}$ but no point of $Y\left(P_{1}\right)-c_{2}$ which is such that $J_{2}-P_{1}-P_{2}$ contains no point of $Y^{*}$ and is inclosed by $O$. This process is continued until for each $c_{i}$ there is a unique $J_{i}$ inclosing it.

The interior of $P_{1} A P_{2}+P_{1} B P_{2}$ is topologically equivalent to the bounded complementary domain of $P_{1} B P_{2}+P_{2} C+P_{1} D P_{2}$. Treating $P_{2} B P_{1}+P_{1} D P_{2}$ as $P_{1} A P_{2}$ and $P_{2} C+C P_{2}$ as $P_{1} B P_{2}$, we consider curves $J_{1}^{\prime}, J_{2}^{\prime}, \cdots$ inclosing the components of $y\left(P_{2}\right)-P_{2}$ in a manner similar to that in which we considered simple closed curves inclosing the components of $y\left(P_{1}\right)-P_{1}$.

Let $g_{1}^{\prime}$ denote the closure of $J+P_{1} B P_{2}+P_{2} C+J_{1}+J_{2}+\cdots+J_{1}^{\prime}+J_{2}^{\prime}$ $+\cdots$. The continuum $g_{1}^{\prime}$ is a subset of an element of $G^{\prime}$. It is to be noted that $g_{1}^{\prime}$ is a continuum of condensation of $M$ intersecting both $V_{5}^{\prime} V_{2}$ and $V_{4} V_{6}$ and if $C$ is the common part of $M$ and a bounded complementary domain of $g_{1}^{\prime}$, then (1) $C$ intersects the interiors of triangles $V_{1} V_{2} V_{5}$ and $V_{4} V_{3} V_{6}$, (2) $\bar{C}$ is a simple plane web, and (3) no two elements of $Y$ intersect both $\dot{C}$ and the boundary of $C$ with respect to $M$.

Second step. Let $i$ be the least positive integer $n$ such that $y_{n}$ intersects one of the intervals $V_{1} P_{1}$ and $V_{4} P_{2}$ in a point $P$ nearer the midpoint than either end point of that interval. If there is no such $i$, let $P$ be the midpoint of $V_{1} P_{1}$. Let $P_{3}, P_{4}$ be the projections of $P$ on $V_{1} P_{1}$ and $V_{4} P_{2}$ respectively. By treating $V_{1} P_{1}+P_{1} A P_{2}+P_{2} V_{4}+V_{4} V_{1}$ as the boundary of $R$ and following a procedure similar to that used in finding $g_{1}^{\prime}$, we obtain $g_{2}^{\prime}$. By treating $P_{1} V_{2}+V_{2} V_{3}+V_{3} P_{2}+P_{2} D P_{1}$ as the boundary of $R$, we get $g_{3}^{\prime}$. This process is continued until every point of $M$ belongs to either the closure of the sum of all the $g_{n}^{\prime \prime}$ 's or to a bounded complementary domain of one of them.

If $U$ is a component of $M$ minus the common part of $M$ and the sum of the $g_{n}^{\prime \prime}$ 's together with their bounded complementary domains and $U$ is closed, $U$ will have a point in common with $V_{1} V_{2}$ and a point in common with $V_{4} V_{3}$ 
and is to be considered as a $g^{\prime}$. If $U$ is not closed, it will not have a point in common with either $V_{1} V_{2}$ or $V_{4} V_{3}$ and, as every two $g_{n}{ }^{\prime}$ 's are separated in $M$ by a third $g_{n}^{\prime}$, there is only one $g_{n}^{\prime}$ which contains a limit point of $U$. If there are such $U^{\prime}$ 's, $g_{n}^{\prime}$ plus all $U^{\prime}$ 's which have a point of $g_{n}^{\prime}$ as a limit point is a $g^{\prime}$. Each $g^{\prime}$ described is a subset of an element of $G^{\prime}$.

If $G_{1}^{\prime}$ is the set of maximal $g^{\prime \prime}$ 's thus described, $G_{1}^{\prime}$ is an arc with respect to its elements, each point of $G_{1}^{\prime *}$ is a limit point of $M^{\prime}-G_{1}^{\prime *}$, no element of $G_{1}^{\prime}$ has two points in common with an element of $Y$ and each element of $G_{1}^{\prime}$ has both a point in common with $V_{1} V_{2}$ and a point in common with $V_{4} V_{3}$. There exists a dendron $F_{1}^{\prime}$ in $M$ such that $F_{1}^{\prime} \cdot G_{1}^{\prime *}$ is $V_{4} V_{3}, F_{1}^{\prime}-V_{4} V_{3}$ intersects neither $V_{4} V_{6}$ nor any element of $Y$ and if $C$ is a component of $M-G_{1}^{\prime *}$, then $F_{1}^{\prime} \cdot C$ is an arc minus an end point of diameter less than the diameter of $V_{1} V_{2}$ and if $K$ is a connected subset of $C$ that intersects both this arc and an element of $Y$ that intersects the boundary of $C$ with respect to $M$, then $K$ intersects both $V_{6} V_{2}$ and $V_{4} V_{6}$. Let $D_{1}$ be a domain containing $F_{1}^{\prime}-V_{4} V_{3}$ such that each point of $D_{1}$ is nearer $F_{1}^{\prime}$ than $G_{1}^{\prime *}+V_{4} V_{6}$. Then $\bar{D}_{1}$ intersects $G_{1}^{\prime *}$ only in $V_{4} V_{3}$.

Third step. If $C$ is a component of $M-G_{1}^{*}, C$ is the common part of $M$ and a bounded complementary domain of some element of $G_{1}^{\prime}$. We now consider the breaking up of $\bar{C}$ into elements of $G^{\prime}$. We note that $C$ intersects the interiors of the triangles $V_{1} V_{2} V_{6}$ and $V_{4} V_{3} V_{6}$. If $\beta$ denotes the boundary of $C$ with respect to $M$, then $C+\beta$ is a simple plane web and no more than one element of $Y$ intersects both $C$ and $\beta$.

Let $y_{a}$ be such an element of $Y$ intersecting $\beta$ at $P_{1}$ and let $c$ denote the closure of the common part of $y_{a}$ and $C$. Let $Q_{1} Q_{2}$ be an arc such that $Q_{1} Q_{2}-Q_{1}$ is $F_{1}^{\prime} \cdot C$. Let $i$ be the least positive integer $n$ such that $d_{n}$ is a subset of $c$. There is a point $P_{2}$ of the boundary of $d_{i}$ such that $c+\beta-P_{2}$ is connected. There is a subcontinuum $w$ of $c$ such that (1) $w$ contains $P_{1}$ and $P_{2},(2)$ if a point of a complementary domain of $M$ belongs to $w$, the complementary domain is a subset of $w$, and (3) no subcontinuum of $w$ has properties (1) and (2). There is an upper semi-continuous collection $E$ of mutually exclusive continua filling up $w$ such that $E$ is an arc from $P_{1}$ to $P_{2}$ with respect to its elements and such that every element of $E$ is either a point or an arc which has only its end points in common with the boundary of $c$. Designate the arc of elements of $E$ by $\xi\left(P_{1} P_{2}\right)$. Each connected subset of $C$ that intersects $\xi\left(P_{1} P_{2}\right)$ and $Q_{1} Q_{2}$ also intersects $V_{6} V_{2}$ and $V_{4} V_{6}$. To adjust the argument to the case where no element of $Y$ has a point in common with both $C$ and $\beta$, let $\xi\left(P_{1} P_{2}\right)$ be an arc in $C+P_{1}$ from a point $P_{1}$ of $\beta$ to a point $P_{2}$ of $C$ such that $\xi\left(P_{1} P_{2}\right)$ contains no point of $Y^{*}$ and any connected subset of $C$ intersecting both $\xi\left(P_{1} P_{2}\right)$ and $Q_{1} Q_{2}$ also intersects both $V_{5} V_{2}$ and $V_{4} V_{6}$.

Let $j, k$ be the least positive integers $m, n$ respectively such that $z_{m}, y_{n}$ are subsets of $C$. There is a simple closed curve $J$ lying in $C$, inclosing $y_{k}$, intersecting $\xi\left(P_{1} P_{2}\right)+Q_{1} Q_{2}$ only at $P_{2}+Q_{2}$ and such that $Q_{1} Q_{2}+J$ contains $z_{j}$. 
We note that $J$ plus the common part of $M$ and the interior of $J$ is a simple plane web intersecting both $V_{5} V_{2}$ and $V_{4} V_{6}$.

A breaking up of the portion of $\bar{C}$ not in the interior of $J$ into elements of $G^{\prime}$ will now be considered. Considering the elements of $\xi\left(P_{1} P_{2}\right)$ as points, $\xi\left(P_{1} P_{2}\right)$ as $V_{1} V_{2}, \beta$ as $V_{1} V_{4}, \xi\left(P_{1} P_{2}\right)$ as $V_{4} V_{3}, J$ as $V_{2} V_{3}$, and $Q_{1} Q_{2}$ as $V_{5} V_{6}$, we divide the portion of $\bar{C}$ not in the interior of $J$ in a manner somewhat analogous to that described in the second step. We divide the portion of $\bar{C}$ corresponding to $V_{1} V_{2} V_{6} V_{5}$ in the manner in which $V_{1} V_{2} V_{3} V_{4}$ was divided in the second step and we divide the portion of $\bar{C}$ corresponding to $V_{5} V_{6} V_{3} V_{4}$ in the same fashion, care being taken that each pair of $g$ "s thus defined that intersect on $Q_{1} Q_{2}$ also intersect the same elements of $\xi\left(P_{1} P_{2}\right)$.

The closures of all components of $M-G_{1}^{\prime *}$ are broken up just as was $\bar{C}$. If a $g^{\prime}$ as defined in this step intersects another $g^{\prime}$ defined in either this or the second step, the sum of the two is a $g^{\prime}$. Let $G_{2}^{\prime}$ denote the set of maximal $g^{\prime \prime}$ s defined. We note that the boundary of $d_{i}$ of $y_{a}$ is a subset of $G_{2}^{\prime *}$. Also, each element of $G_{2}^{\prime}$ intersects $V_{5} V_{2}$ and $V_{4} V_{6}$ and so does each component of $M-G_{2}^{\prime *}$.

There exists a dendron $F_{2}^{\prime}$ in $M$ such that $F_{2}^{\prime} \cdot G_{2}^{\prime *}$ is $F_{1}^{\prime}, F_{2}^{\prime}-F_{1}^{\prime}$ is a subset of $D_{1}, F_{2}^{\prime}-F_{1}^{\prime}$ intersects no element of $Y$, and if $C$ is a component of $M-G_{2}^{\prime *}$, then $F_{2}^{\prime} \cdot C$ is an arc minus one end point of diameter less than onehalf the diameter of $V_{1} V_{2}$. Let $D_{2}$ be a domain containing $F_{2}^{\prime}-F_{1}^{\prime}$ such that each point of $D_{2}$ is nearer $F_{2}^{\prime}$ than $G_{2}^{\prime *} \bar{D}_{2}$ and is a subset of $D_{1}+F_{1}^{\prime}$.

Fourth step. We shall now describe the breaking up of a component $C$ of $M-G_{2}^{\prime *}$. The boundary of $C$ with respect to $M$ is a simple closed curve $J$. If an element of $Y$ intersects both $C$ and $J$, break up $\bar{C}$ as described in the third step. Otherwise, let $j$ be the least integer equal to $n$ such that $y_{n}$ is inclosed by $J$. Let $P$ be a point of $y_{j}$ such that $y_{j}-P$ is connected. Let $Q_{1} Q_{2}-Q_{1}$ be the common part of $F_{2}^{\prime}$ and the interior of $J$. There is an arc $Q_{1} P$ in $M$ which except for its end points intersects neither $J+Q_{1} Q_{2}$ nor any element of $Y$ and which is such that any connected subset of the interior of $J$ intersecting $Q_{1} Q_{2}$ and $y_{j}$ but not $Q_{1} P$ intersects both $V_{5} V_{2}$ and $V_{4} V_{6}$. Treating $Q_{1} P+J$, $Q_{1} Q_{2}$, and $y_{j}$ as $\beta, Q_{1} Q_{2}$, and $c$ respectively were treated in the third step, we break up the common part of $M$ and the interior of $J$ in a manner analogous to that in which $C$ was broken up as considered in the third step.

Let $G_{3}^{\prime}$ be the set of all $g^{\prime \prime}$ s considered after all components of $M-G_{2}^{\prime *}$ are broken up as described above. We define a dendron $F_{3}^{\prime}$ in $D_{2}$ containing $F_{2}^{\prime}$ and satisfying conditions analogous to those satisfied by $F_{2}^{\prime}$. In like manner, we consider $G_{4}^{\prime}, G_{5}^{\prime}, \cdots$ and $F_{4}^{\prime}, F_{5}^{\prime}, \ldots$ It is to be noted that (1) for each positive integer $j$, there is an $n$ such that $z_{j}$ and the boundary of $d_{i}$ belongs to $G_{n}{ }^{*}$, (2) for no $n$ do three points of $Y^{*}$ belong to the same element of $G_{n}^{\prime}$, (3) each element of $G_{n}^{\prime}$ intersects $V_{5} V_{2}$ and $V_{4} V_{6}$, and (4) each component of $M-G_{n}^{\prime *}$ intersects $V_{5} V_{2}$ and $V_{4} V_{6}$.

Definition of dendron $G$. Let $G^{\prime}$ be the set of all continua such that $g^{\prime}$ 
is an element of $G^{\prime}$ only if either (1) for some positive integer $n, g^{\prime}$ belongs to $G_{n}^{\prime}$ and $G_{n+1}^{\prime}$ or (2) $g^{\prime}$ is a component of $M-\left(G_{1}^{\prime *}+G_{2}^{\prime *}+\cdots\right)$. With respect to its elements, $G^{\prime}$ is a dendron. Each element of $G^{\prime}$ is a continuum intersecting both $V_{5} V_{2}$ and $V_{4} V_{6}$. Each element of $G^{\prime}$ is a continuum of condensation of $M$. No element of $G^{\prime}$ intersects an element of $Y$ in more than two points.

We define $G$ to be the collection of all point sets $g$ such that $g$ is the image of an element of $G^{\prime}$ under $T$. We note that $G$ is an upper semi-continuous collection of mutually exclusive continua filling up $W$ such that $G$ is a dendron with. respect to its elements.

Definition of dendron $F$. We define $F^{\prime}$ to be the limiting set of $F_{1}^{\prime}, F_{2}^{\prime}, \cdots$. We note that it is a dendron that intersects each element of $G^{\prime}$ in only one point. We define $F$ to be the image of $F^{\prime}$ under transformation $T$. We note that $F$ is a dendron that intersects each element of $G$ in only one point and the outer boundary of $W$ in only an arc.

Description of arc $H$. We shall describe an upper semi-continuous collection $H$ of mutually exclusive continua filling up $W$ such that $H$ is an arc with respect to its elements and such that if $g$ and $h$ are elements of $G$ and $H$ respectively, then $g \cdot h$ is totally disconnected. First, we shall establish four additional theorems.

THEOREM 9. If $R$ is the interior of a square and $E$ is a collection of point sets covering $\bar{R}$ such that for each point $P$ and each domain $D$ there is a point $P^{\prime}$ of $D$ which belongs to no element of $E$ that contains $P$, then for any positive $\epsilon$ and any two points $A$ and $B$ of $\bar{R}$ there is in $R+A+B$ an arc $A C B$ such that (1) for any two points $Q_{1}$ and $Q_{2}$ of $A C B\left({ }^{7}\right), \cos \left(Q_{1} Q_{2}, A B\right)>1-\epsilon$ and (2) no element of $E$ contains a subarc of $A C B$.

Proof. There is a point $P_{1}$ such that $\left.{ }^{8}\right) d\left(A, P_{1}\right)=d\left(P_{1}, B\right)$, the intervals $A P_{1}$ and $P_{1} B$ are subsets of $R+A+B$, and $\cos \left(A P_{1}, A B\right)>1-\epsilon$. There exists a quadrilateral $Q$ lying in $R+A+B$ and inclosing $A P_{1}+P_{1} B-(A+B)$. Within $Q$ there exist (1) a point $P_{2}$ not belonging to any element of $E$ that contains $A$ and such that $\max \left[d\left(A, P_{2}\right) ; d\left(P_{2}, P_{1}\right)\right]<(2 / 3) d\left(A, P_{1}\right)$, $\cos \left(A P_{2}, A B\right)>1-\epsilon$ and $\cos \left(P_{2} P_{1}, A B\right)>1-\epsilon$ and (2) a point $P_{3}$ not belonging to any element of $E$ that contains $P_{1}$ and such that $\max \left[d\left(P_{1}, P_{3}\right) ; d\left(P_{3}, B\right)\right]$ $<(2 / 3) d\left(P_{1}, B\right), \cos \left(P_{1} P_{3}, A B\right)>1-\epsilon$ and $\cos \left(P_{3} B, A B\right)>1-\epsilon$. Likewise we get within $Q$ a point $P_{4}$ not belonging to any element of $E$ containing $A$ and such that $\max \left[d\left(A, P_{4}\right) ; d\left(P_{4}, P_{2}\right)\right]<(2 / 3) d\left(A, P_{2}\right)$ and $\cos \left(A P_{4}, A B\right)>1-\epsilon$ and $\cos \left(P_{4} P_{2}, A B\right)>1-\epsilon$. This process is continued.

Let $L$ denote the closure of the sum of all such $P_{n}$ 's. Since $L$ is closed and compact and as its projection on the line $A B$ is the interval $A B$ and each line

$\left.{ }^{7}\right) \mathrm{By} \cos (A B, C D)$ is meant the cosine of the smaller angle between the lines $A B$ and $C D$. If $A B$ and $C D$ are coincident or parallel, $\cos (A B, C D)=1$.

( $\left.{ }^{8}\right) \mathrm{By} d(A, B)$ is meant the distance between $A$ and $B$. 
perpendicular to this interval contains only one point of $L$, then $L$ is an arc from $A$ to $B$.

As each of the numbers $\cos \left(P_{2} P_{1}, A B\right)$ and $\cos \left(P_{1} P_{3}, A B\right)$ is greater than $1-\epsilon$ and as the projection of $P_{1}$ on $A B$ is between the projections of $P_{2}$ and $P_{3}$, it follows that $\cos \left(P_{2} P_{3}, A B\right)>1-\epsilon$. By induction, we get for different integers $j$ and $k$ that $\cos \left(P_{j} P_{k}, A B\right)>1-\epsilon$. Hence if $Q_{1}$ and $Q_{2}$ are points of the continuum $L, \cos \left(Q_{1} Q_{2}, A B\right)>1-\epsilon$.

Each subarc of $L$ contains a $P_{j}$ and a $P_{k}$ such that $P_{j}$ does not belong to any element of $E$ containing $P_{k}$. Hence, no subarc of $L$ is a subset of an element of $E$.

THEOREM 10. Let $R$ be the interior of a square with diagonals $V_{1} V_{3}$ and $V_{2} V_{4}$; let $E$ be a collection of mutually exclusive point sets filling up $\bar{R}$ such that (1) no element of $E$ contains a nondegenerate connected subset of $V_{1} V_{4}+V_{2} V_{3}$ and (2) no element of $E$ is dense in a domain; then there exists an upper semicontinuous collection $H$ of mutually exclusive arcs filling up $\bar{R}$ such that $H$ is an arc with respect to its elements, each element of $H$ has one end point on $V_{1} V_{2}$ and one on $V_{4} V_{3}$, each point of $V_{1} V_{2}+V_{4} V_{3}$ is an end point of an element of $H$; and no element of $E$ contains a subarc of an element of $H$.

Proof. With the help of the preceding theorem, it will be shown that there exists a set of mutually exclusive arcs $\alpha_{1}, \alpha_{2}, \ldots$ such that (1) each of them has one end point on $V_{1} V_{2}$ and the other on $V_{4} V_{3}$ and has no arc in common with an element of $E$ and (2) the components of $\bar{R}-\left(\alpha_{1}+\alpha_{2}+\cdots\right)$ are arcs having one end point on $V_{1} V_{2}$ and one on $V_{4} V_{3}$ and, furthermore, no such component has an arc in common with an element of $E$.

We shall assume that $\bar{R}-R$ is a unit square. Consider points $Q_{1}, Q_{2}, Q_{3}$, $Q_{4}, Q_{5}, Q_{6}$ in the orders $V_{1} Q_{1} Q_{2} Q_{3} V_{4}$ and $V_{2} Q_{4} Q_{5} Q_{6} V_{3}$ and such that $Q_{1}, Q_{2}, Q_{3}$ and $Q_{4}^{4}, Q_{5}, Q_{6}$ divide $V_{1} V_{4}$ and $V_{2} V_{3}$ respectively into four equal parts. Choose a positive number $\epsilon$ less than 1 . Let $P_{0}$ be the midpoint of $V_{1} V_{2}$ and $P_{1}, P_{2}$ be points of $V_{1} V_{2}$ at a distance from $P_{0}$ equal to $\epsilon / 6$ and in the order $V_{1} P_{1} P_{0} P_{2} V_{2}$. Let $P_{3}$ and $P_{4}$ denote points on $V_{1} V_{2}$ at a distance from $P_{0}$ equal to $2 \epsilon / 6$ and in the order $V_{1} P_{3} P_{0} P_{4} V_{2}$. Similarly, consider points $P_{5}, P_{6}, \cdots, P_{2 j-1}, P_{2 j}$ where $P_{2 j-1}$ and $P_{2 j}$ are at a distance from $P_{0}$ equal to $j \epsilon / 6$ and from $V_{1}$ and $V_{2}$ respectively of not more than $\epsilon / 6$ and are in the order $V_{1} P_{2 j-1} P_{0} P_{2 j} V_{2}$. Let $P_{2 j+1}$ be the midpoint of $V_{1} P_{2 j-1}$ and in general let $P_{2 j+2 k+1}$ be the midpoint of $V_{1} P_{2 j+2 k-1}$ for all positive integers $k$. Also $P_{2 j+2 k+2}$ is the midpoint of $P_{2 j+2 k} V_{2}$. Let $P_{i, 1}, P_{i, 2}, P_{i, 3}, P_{i, 4}$ be the projections of $P_{i}$ on $Q_{1} Q_{4}, Q_{2} Q_{5}, Q_{3} Q_{6}, V_{4} V_{3}$ respectively.

It is assumed that each of the following arcs has the properties that (1) no subarc of it is a subset of $E$ and (2) if $A$ and $B$ are two points of it, $\cos \left(A B, V_{1} V_{4}\right)>1 /\left(1+\epsilon^{2}\right)^{1 / 2}$. There exist in $P_{0}+P_{2}+R$ mutually exclusive arcs $P_{0} P_{0,1}$ and $P_{2} P_{2,1}$ satisfying the above two conditions and such that no arc from $P_{0} P_{2}$ to $P_{0,1} P_{2,1}$ in $P_{0} P_{2}+P_{0,1} P_{2,1}$ plus the interior of $P_{0} P_{2}+P_{2} P_{2,1}$ 
$+P_{2,1} P_{0,1}+P_{0,1} P_{0}$ is a subset of an element of $E$. In the case where the elements of $E$ contain arcs, this may be seen by constructing $P_{0} P_{0,1}$ and $P_{2} P_{2,1}$ so that two elements of $E$ contain arcs from $P_{0} P_{0,1}$ to $P_{2} P_{2,1}$ in $P_{0} P_{0,1}+P_{2} P_{2,1}$ plus the interior of $P_{0} P_{2}+P_{2} P_{2,1}+P_{2,1} P_{0,1}+P_{0,1} P_{0}$. Also, there exist in $R$ mutually exclusive arcs $P_{0,2} P_{0,3}$ and $P_{2,2} P_{2,3}$ which satisfy the above two conditions and such that no arc from $P_{0,2} P_{2,2}$ to $P_{0,3} P_{2,3}$ in $P_{0,2} P_{2,2}+P_{0,3} P_{2,3}$ plus the interior of $P_{0,2} P_{2,2}+P_{2,2} P_{2,3}+P_{2,3} P_{0,3}+P_{0,3} P_{0,2}$ is a subset of an element of $E$. Furthermore, there are mutually exclusive arcs $P_{0,1} P_{0,2}$ and $P_{1,1} P_{1,2}$ in $R$ satisfying the above two conditions and such that no arc from $P_{1,1} P_{0,1}$ to $P_{1,2} P_{0,2}$ in $P_{1,1} P_{0,1}+P_{1,2} P_{0,2}$ plus the interior of $P_{1,1} P_{0,1}+P_{0,1} P_{0,2}$ $+P_{0,2} P_{1,2}+P_{1,2} P_{1,1}$ is a subset of an element of $E$. Arcs satisfying corresponding conditions join $P_{1,3}, P_{0,3}$ to $P_{1,4}, P_{0,4}$ respectively. Furthermore, arcs which intersect no arc previously described and which satisfy conditions analogous to those given join $P_{3}, P_{1}$ to $P_{3,1}, P_{1,1} ; P_{3,2}, P_{1,2}$ to $P_{3,3}, P_{1,3} ; P_{2,1}, P_{4,1}$ to $P_{2,2}, P_{4,2} ; P_{2,3}, P_{4,3}$ to $P_{2,4}, P_{4,4} ; P_{5,1}, P_{3,1}$ to $P_{5,2}, P_{3,2} ; \cdots$ in such a manner that if $\alpha_{i}$ designates $P_{i} P_{i, 1}+P_{i, 1} P_{i, 2}+P_{i, 2} P_{i, 3}+P_{i, 3} P_{i, 4}$, then $V_{1} V_{4}, V_{2} V_{3}$ are the sequential limiting sets of $\alpha_{1}, \alpha_{3}, \cdots$ and $\alpha_{0}, \alpha_{2}, \cdots$ respectively.

Let $H_{1}$ be the set consisting of $V_{1} V_{4}, V_{2} V_{3}, \alpha_{0}, \alpha_{1}, \ldots$ If $h_{1}$ and $h_{2}$ are elements of $H_{1}$ which are not separated from each other in $\bar{R}$ by any element of $H_{1}$ and $C$ is set of all points of $\bar{R}$ between $h_{1}$ and $h_{2}$, no arc from $Q_{2} Q_{5}$ to $V_{1} V_{2}+V_{4} V_{3}$ in $Q_{2} Q_{5}+V_{1} V_{2}+V_{4} V_{3}$ plus the bounded complementary domain of $V_{1} V_{2}+V_{4} V_{3}+h_{1}+h_{2}$ is a subset of an element of $E$.

Designate $V_{1} V_{2} \cdot \bar{C}$ and $V_{4} V_{3} \cdot \bar{C}$ by $U_{1} U_{2}$ and $U_{4} U_{3}$ respectively, where the points $U_{1}, U_{2}, U_{3}, U_{4}$ are in the orders $V_{1} U_{1} U_{2} V_{2}$ and $V_{4} U_{4} U_{3} V_{3}$. Treating $U_{1} U_{2}$ and $U_{4} U_{3}$ as $V_{1} V_{2}$ and $V_{4} V_{3}$ were treated, we break up $\bar{C}$ in a manner somewhat analogous to that in which $\bar{R}$ was divided except that instead of three lines $Q_{1} Q_{4}, Q_{2} Q_{5}, Q_{3} Q_{6}$, we use seven lines parallel to $U_{1} U_{2}$ that divide a segment from $U_{1} U_{2}$ to $U_{4} U_{3}$ into eight equal parts. The division of all such $C$ 's gives us $H_{2}$. This division of $C$ is possible, for no use was made above of the fact that $V_{1} V_{4}$ and $V_{2} V_{3}$ were straight lines. In fact; it can be shown that if (1) $P_{1} P_{2}$ and $P_{1}^{\prime} P_{2}^{\prime}$ are parallel intervals, (2) $P_{1} P_{1}^{\prime}$ and $P_{2} P_{2}^{\prime}$ are mutually exclusive arcs such that if $A$ and $B$ are either two points of $P_{1} P_{1}^{\prime}$ or two points of $P_{2} P_{2}^{\prime}$, then $\sin \left(A B, P_{1} P_{2}\right)>1 /\left(1+\epsilon^{2}\right)^{1 / 2}$, (3) $Q_{1}, Q_{2}, Q_{1}^{\prime}, Q_{2}^{\prime}$ are points in the orders $P_{1} Q_{1} Q_{2} P_{2}$ and $P_{1}^{\prime} Q_{1}^{\prime} Q_{2}^{\prime} P_{2}^{\prime}$ and $\min$ [sin $\left(Q_{1} Q_{2}^{\prime}, P_{1} P_{2}\right)$; $\left.\sin \left(Q_{2} Q_{1}^{\prime}, P_{1} P_{2}\right)\right]>1 /\left(1+\epsilon^{2}\right)^{1 / 2}$, and (4) $E$ is a collection of mutually exclusive point sets filling up $P_{1} P_{2}+P_{2} P_{2}^{\prime}+P_{2}^{\prime} P_{1}^{\prime}+P_{1}^{\prime} P_{1}$ plus its interior and such that no element of $E$ is dense in a domain, then there exist in its interior plus the points $Q_{1}, Q_{2}, Q_{1}^{\prime}$ and $Q_{2}^{\prime}$ two mutually exclusive arcs $Q_{1} Q_{1}^{\prime}$ and $Q_{2} Q_{2}^{\prime}$ such that no arc from $Q_{1} Q_{2}$ to $Q_{1}^{\prime} Q_{2}^{\prime}$ in $Q_{1} Q_{2}+Q_{2} Q_{2}^{\prime}+Q_{2}^{\prime} Q_{1}^{\prime}+Q_{1}^{\prime} Q_{1}$ plus its interior is a subset of an element of $E$ and such that if $C$ and $D$ are two points of- $Q_{1} Q_{1}^{\prime}$ or two points of $Q_{2} Q_{2}^{\prime}$, then $\sin \left(C D, P_{1} P_{2}\right)>1 /\left(1+\epsilon^{2}\right)^{1 / 2}$.

No element of $E$ contains an arc in $\bar{R}-\left(H_{1}^{*}+H_{2}^{*}\right)$ whose projection of $V_{1} V_{4}$ is of length $3 / 8$. With the use of fifteen lines parallel to $V_{1} V_{2}, H_{3}$ is de- 
fined in such a way that no element of $E$ contains an arc in $\bar{R}-\left(H_{1}^{*}+H_{2}^{*}+H_{3}^{*}\right)$ whose projection on $V_{1} V_{4}$ is of length $3 / 16$. If a suitable pattern is used in defining $H_{4}, H_{5}, \cdots$ and $H$ is the set such that $h$ is an element of $H$ only if it is an element of some $H_{n}$ or a component of $\bar{R}-\left(H_{1}^{*}+H_{2}^{*}+\cdots\right)$, then $H$ satisfies the conditions of our theorem.

Our method actually gives the following result.

THEOREM 11. Let $R$ be the interior of a square with diagonals $V_{1} V_{3}$ and $V_{4} V_{2}$; let $E$ be a collection of point sets covering $\bar{R}$ such that no element of $E$ contains a nondegenerate connected subset of $V_{1} V_{4}+V_{2} V_{3}$ and if $D_{1}$ and $D_{2}$ are two domains, there exist nondegenerate connected subsets $f_{1}$ and $f_{2}$ of $D_{1}$ and $D_{2}$ respectively such that no element of $E$ intersects both $f_{1}$ and $f_{2}$. Then there exists an upper semi-continuous collection $H$ of mutually exclusive arcs filling up $\bar{R}$ such that $H$ is an arc with respect to its elements, each element of $H$ has one end point on $V_{1} V_{2}$ and one on $V_{4} V_{3}$, each point of $V_{1} V_{2}+V_{4} V_{3}$ is an end point of an element of $H$ and no element of $E$ contains a subarc of an element of $H$.

THEOREM 12. Let $R$ be the interior of a square with diagonals $V_{1} V_{3}$ and $V_{2} V_{4}$; let $F$ be a dendron in $\bar{R}$ intersecting the boundary of $R$ only in $V_{4} V_{3}$; let $E$ be a collection of point sets covering $\bar{R}$ such that no element of $E$ contains a nondegenerate connected subset of $V_{1} V_{4}+V_{2} V_{3}$ and if $D_{1}$ and $D_{2}$ are two domains, there exist nondegenerate connected subsets $f_{1}$ and $f_{2}$ in $D_{1}$ and $D_{2}$ respectively such that no element of $E$ intersects both $f_{1}$ and $f_{2}$. There exists an upper semi-continuous collection $H$ of mutually exclusive continua filling up $\bar{R}$ such that $H$ is a dendron with respect to its elements, $V_{1} V_{4}$ is a subset of an element of $H$ and so is $V_{2} V_{3}$, no element of $E$ contains a nondegenerate subcontinuum of an element of $H$ and each element of $H$ intersects $F$ in only one point and $V_{1} V_{2}$ in only a totally disconnected point set.

Indication of proof. We construct dendron $H$ so that each element of $H$ intersects $F$ in only one point in a manner analogous to that in which we constructed $G^{\prime}$ so that it intersected $F^{\prime}$ in only one point. We use the methods of Theorem 10 to insure that no element of $H$ intersects an element of $E$ in a nondegenerate continuum.

Definition of arc $H$. We shall now define an upper semi-continuous collection $H$ of mutually exclusive continua filling up $W$ such that $H$ is an arc with respect to its elements, each element of $H$ intersects each element of $G$, and the intersection of an element of $G$ and an element of $H$ is a totally disconnected point set.

As the points of $M-M \cdot Y^{*}$ and the elements of $Y$ form an upper semicontinuous collection of mutually exclusive continua filling up $\bar{R}$, it follows [5] that there is a continuous transformation $T^{\prime}$ carrying $M+Y^{*}$ into $\bar{R}$ such that (1) $T^{\prime}$ carries each point of $V_{5} V_{2}+V_{4} V_{6}$ plus the portion of the boundary of $R$ not belonging to $Y^{*}$ into itself, (2) $T^{\prime}$ carries each point of $M-M \cdot Y^{*}$ 
into a point, (3) $T^{\prime}$ carries each element of $Y$ into a point, and (4) each point of $\bar{R}$ is either the image of only one point of $M-M \cdot Y^{*}$ or the image of only one element of $Y$. If $G^{\prime \prime}$ is the set of all $T^{\prime}\left(g^{\prime}\right)$ 's where $g^{\prime}$ is an element of $G^{\prime}$, the elements of $G^{\prime \prime}$ intersect $V_{5} V_{2}$ and $V_{4} V_{6}$. By the methods of Theorem 11, it can be shown that there is an upper semi-continuous collection $H^{\prime \prime}$ of mutually exclusive continua filling up $\bar{R}$ such that (1) $H^{\prime \prime}$ is with respect to its elements an arc with ends at $V_{1}$ and $V_{3}$, (2) every element of $H^{\prime \prime}$ other than $V_{1}$ and $V_{2}$ is an arc having only one point on $V_{1} V_{2}+V_{2} V_{3}$ and only one point on $V_{1} V_{4}+V_{4} V_{3}$, these points being its end points, (3) if $g^{\prime \prime}$ is an element of $G^{\prime \prime}$ intersecting an element $h^{\prime \prime}$ of $H^{\prime \prime}, g^{\prime \prime} \cdot h^{\prime \prime}$ is totally disconnected, and (4) one element of $H^{\prime \prime}$ contains $V_{5}+V_{2}$ and another contains $V_{4}+V_{6}$ and each of these elements lies except for its end points on the interior of the parallelogram $V_{5} V_{2} V_{6} V_{4}$.

If $H^{\prime}$ is a set such that $h^{\prime}$ is an element of $H^{\prime}$ only if it is a subcontinuum of $M$ which is maximal with respect to $T^{\prime}\left(h^{\prime}\right)$ being an element of $H^{\prime \prime}$, then $H^{\prime}$ is a collection of mutually exclusive continua filling up $M$. Furthermore, it is an arc with respect to its elements. As no element of $G^{\prime}$ has more than two points in common with the boundary of an element of $Y$, if $g^{\prime}$ is an element of $G^{\prime}$ having a point in common with an element $h^{\prime}$ of $H^{\prime}, g^{\prime} \cdot h^{\prime}$ is totally disconnected.

Let $H$ be a set such that $h$ is an element of $H$ only if it is a continuum which is maximal with respect to being the image under $T$ of the sum of two or three elements of $H^{\prime}$. Then $H$ is the required collection of mutually exclusive continua filling up $W$. The sufficiency of Theorem 1 is demonstrated.

Theorem 1. may also be stated as follows:

Theorem 1. A necessary and sufficient condition that a compact plane continuum $W$ remain connected and locally connected on the omission of any countable subset is that there exist two continuous monotone transformations $T_{1}$ and $T_{2}$ such that both $T_{1}(W)$ and $T_{2}(W)$ are nondegenerate dendrons and if $a$ and $b$ are elements of $T_{1}(W)$ and $T_{2}(W)$ respectively, then $T_{1}^{-1}(a) \cdot T_{2}^{-1}(b)$ exists and is totally disconnected $\left({ }^{9}\right)$.

Alternate definition of a simple plane web. Actually, we have shown that the following is an equivalent definition to that previously given.

Definition. A compact plane continuum $W$ is a simple plane web provided there exist an upper semi-continuous collection $G$ of mutually exclusive continua filling up $W$ and another such collection $H$ also filling up $W$ such that (1) $G$ is a dendron with respect to its elements and $H$ is an arc with respect to its elements and (2) if $g$ and $h$ are elements of $G$ and $H$ respectively, then $g \cdot h$ exists and is totally disconnected. It has been shown [1] that condition (1) is unnecessary.

( ${ }^{9}$ G. T. Whyburn and some of his students have worked with monotone mappings onto dendrons. See [9] for references. 
However, we cannot substitute for (1) in the above definition the condition $\left(1^{\prime}\right)$ that $G$ is an arc with respect to its elements and so is $H$. Consider the plane continuum $W$ obtained by subtracting from a circle $C_{0}$ plus its interior the interiors of five mutually exclusive circles $C_{1}, C_{2}, C_{3}, C_{4}, C_{5}$, each of which is tangent to $C_{0}$ internally. Now $C_{i}$ is not both a subset of an element of $G$ and also a subset of an element of $H$. Assume that $C_{j}(j=1,2,3)$ is not a subset of an element of $G$. Then there are uncountably many elements of $G$ that intersect $C_{j}$. It may be seen that some element $g_{j}$ of $G$ separates a point $P_{i}$ of $C_{j}$ from $C_{0}+C_{j}+\cdots+C_{5}-C_{j}$ in $W$ and intersects $C_{i}(i=0,1, \cdots, 5)$ only if $i$ is equal to $j$. No one of the elements $g_{1}, g_{2}, g_{3}$ separates the other two from each other in $W$. Hence, $G$ is not an arc with respect to its elements.

Theorem 13. If $W$ is a simple plane web, there exist a dendron $F$ in $W$, an arc $\alpha$ in $F$, an upper semi-continuous collection $G$ of mutually exclusive continua filling up $W$ and another such collection $H$ also filling up $W$ such that (1) $G$ is a dendron with respect to its elements and $H$ is an arc with respect to its elements, (2) if $g$ and $h$ are elements of $G$ and $H$ respectively, $g \cdot h$ exists and is totally disconnected, (3) each element of $G$ intersects $F$ in only one point, and (4) each element of $H$ intersects $\alpha$ in only one point.

Proof. Consider the dendron $F$ and the upper semi-continuous collections $G$ and $H$ already defined. If $\alpha$ is the common part of $F$ and the outer boundary of $W$, then $F, \alpha, G$, and $H$ satisfy the conditions of our theorem.

THEOREм 14. If $W$ is a simple plane web, there exist a dendron $F$ in $W$, an upper semi-continuous collection $G$ of mutually exclusive continua filling up $W$ and another collection $H$ also filling up $W$ such that (1) $G$ is a dendron with respect to its elements and so is $H$, (2) if $g$ and h are elements of $G$ and $H$ respectively, $g \cdot h$ exists and is totally disconnected, (3) each element of $G$ intersects $F$ in only one point and so does each element of $H$.

Proof. The dendron $F$ and the upper semi-continuous collection $G$ are the same as those previously described.

We shall use the same symbols as those used in our definition of the arc $H$. Let $F^{\prime \prime}$ be the image of $F^{\prime}$ under $T^{\prime}$. If $h$ is the element of $H^{\prime \prime}$ containing $V_{4}+V_{6}$, let $H_{1}^{\prime \prime}$ denote the set of all continua $h^{\prime \prime}$ such that $h^{\prime \prime}$ is either $V_{1}$, $h$, or an element of $H^{\prime \prime}$ between $V_{1}$ and $h$. Using the methods of Theorem 12, it can be shown that there exists an upper semi-continuous collection $H_{2}^{\prime \prime}$ of mutually exclusive continua filling up $h+\left(\bar{R}-H_{1}^{\prime \prime}{ }^{*}\right)$ such that (1) $H_{2}^{\prime \prime}$ is a dendron with respect to its elements, (2) $h$ is a subset of an element of $H_{2}^{\prime \prime}$, (3) each element of $H_{2}^{\prime \prime}$ intersects $F^{\prime \prime}$ in only one point and $V_{6} V_{3}$ in a totally disconnected set, and (4) if $g^{\prime \prime}$ is an element of $G^{\prime \prime}$ intersecting an element $h^{\prime \prime}$ of $H_{2}^{\prime \prime}, g^{\prime \prime} \cdot h^{\prime \prime}$ is totally disconnected. We define $H$ from $H_{1}^{\prime \prime}+H_{2}^{\prime \prime}$ in the same manner in which the arc $H$ was defined from $H^{\prime \prime}$.

Theorem 14 may also be stated as follows. 
THEOREM 14. Suppose that $W$ is a compact plane continuum and that $T_{1}, T_{2}$ are continuous monotone transformations of $W$ such that if $a$ and $b$ are elements of $T_{1}(W)$ and $T_{2}(W)$ respectively, then $T_{1}^{-1}(a)$ and $T_{1}^{-2}(b)$ are nondegenerate sets whose common part exists and is totally disconnected. Then there exist monotone retractions $R_{1}$ and $R_{2}$ of $W$ such that $R_{1}(W)$ is a dendron, $R_{2}(W)$ is the same dendron and if $a$ and $b$ are elements of this dendron, $R_{1}^{-1}(a) \cdot R_{2}^{-1}(b)$ exists and is totally disconnected.

\section{REFERENCES}

1. R. H. Bing, Collections filling up a simple plane web, Bull. Amer. Math. Soc. vol. 51 (1945) pp. 674-679.

2. S. Eilenberg, Sur les transformations d'espaces métriques en circonférence, Fund. Math. vol. 24 (1935) pp. 160-176.

3. R. L. Moore, Concerning continua which have dendratomic subsets, Proc. Nat. Acad. Sci. U.S.A. vol. 29 (1943) pp. 384-389.

4. - Concerning continuous curves in the plane, Math. Zeit. vol. 15 (1922) pp. 254-260.

5. - Concerning upper semi-continuous collections of continua, Trans. Amer. Math. Soc. vol. 27 (1925) pp. 416-428.

6. - Concerning webs in the plane, Proc. Nat. Acad. Sci. U.S.A. vol. 29 (1943) pp. 389-393.

7. - Foundations of point set theory, Amer. Math. Soc. Colloquium Publications, vol. 13, New York, 1932.

8. - Bull. Amer. Math. Soc. vol. 33 (1927) p. 141 and vol. 35 (1929) p. 776.

9. G. T. Whyburn, Analytic topology, Amer. Math. Soc. Colloquium Publications, vol. 28, New York, 1942.

10. R. L. Wilder, Concerning continuous curves, Fund. Math. vol. 7 (1925) pp. 340-377.

The University of TeXas 